\title{
miR-297 Protects Human Umbilical Vein Endothelial Cells against LPS-Induced Inflammatory Response and Apoptosis
}

\author{
Yanfen Yao ${ }^{a}$ Haiyan Jia ${ }^{a}$ Geng Wang ${ }^{b}$ Yuezhen Ma ${ }^{a}$ Wei Sun \\ Pibao Lia \\ aDepartment of Intensive Care Unit, Shandong Provincial Third Hospital, Jinan, China, bepartment of \\ Emergency, Jinan City People's Hospital, Jinan, China
}

\section{Key Words}

miR-297 • Inflammation • Apoptosis • HUVECs

\begin{abstract}
Background/Aims: Recently, microRNA-297 (miR-297) and signal transducer and activator of transcription 3 (STAT3) have been demonstrated to be involved in dysfunction of vascular endothelial cells and inflammatory conditions, such as sepsis. The present study aimed to investigate the role of miR-297 and STAT3 in lipopolysaccharide (LPS)-induced inflammatory human umbilical vein endothelial cells (HUVECs). Methods: HUVECs were stimulated by different concentrations of LPS. miR-297 mimics were transfected into HUVECs to overexpress miR-297. The qRT-PCR was used to measure the expression level of miR-297. Western blot was used to detect the expressions of STAT3, inflammatory cytokines, adhesion molecules and apoptosis-related proteins. Cell apoptosis was determined by flow cytometry. Results: Compared with parental HUVECs, the expression of miR-297 was significantly down-regulated, while the expression of STAT3 was obviously up-regulated in LPS-induced HUVECs. The expressions of interleukin- 6 (IL-6), tumor necrosis factor- $\alpha$ (TNF- $\alpha$ ), intercellular adhesion molecule-1 (ICAM-1), vascular cell adhesion molecule-1 (VCAM-1) and E-selectin were also increased in LPS-induced HUVECs than those in parental HUVECs. In addition, LPS induced apoptosis of HUVECs through up-regulation of Bax and cleaved caspase 3 expressions. Conversely, miR-297 mimics inhibited LPS-activated expressions of STAT3, inflammatory cytokines, and adhesion molecules, and protected HUVECs against LPS-induced apoptosis through inhibition of Bax and cleaved caspase 3 expressions. Mechanistically, the 3'-untranslated region (3'-UTR) of STAT3 mRNA was validated as a direct target of miR-297. Over-expression of STAT3 partially abrogated protective effects of miR-297, whereas silencing of STAT3 contributed to miR-297-mediated biological effects. Conclusion: miR-297 protects HUVECs against LPS-induced inflammatory response and apoptosis by targeting STAT3 pathway. Thus, miR-297 may be a promising therapeutic target for patients with sepsis.
\end{abstract}

(C) 2019 The Author(s). Published by

Cell Physiol Biochem Press GmbH\&Co. KG

Pibao Li $\quad$ Department of Intensive Care Unit, Shandong Provincial Third Hospital

No. 12 Central Wuying Hill Road, Jinan 250031, Shandong (China)

E-Mail lipibaoslsy@163.com 


\section{Cellular Physiology Cell Physiol Biochem 2019;52:696-707 \\ \begin{tabular}{ll|l} 
and Biochemistry & $\begin{array}{l}\text { DOl: 10.33594/000000049 } \\
\text { Published online: 29 March 2019 }\end{array}$ & $\begin{array}{l}\text { O 2019 The Author(s). Published by } \\
\text { Cell Physiol Biochem Press GmbH\&Co. KG }\end{array}$ \\
\cline { 2 - 3 } &
\end{tabular} \\ Yao et al.: miR-297 Protects HUVECs}

\section{Introduction}

Sepsis is characterized by acute inflammatory responses to exogenous bacteria and multiple organ injury $[1,2]$, which is one of the leading causes of death among all hospitalized patients in the worldwide. The inflammatory response helps to eliminate pathogens and necrotic cell fragments, and promotes wound healing and tissue regeneration. However, excessive inflammatory mediators would aggravate tissue damage $[3,4]$. Severe sepsis or septic shock can trigger damage to numerous organs, including brain, heart, kidney, liver, and lung [5-7]. Therefore, it is crucial to elucidate the molecular mechanisms underlying inflammatory injury in sepsis.

microRNAs (miRNAs), a kind of non-coding small molecule RNA, are involved in regulation of post-transcriptional gene expressions [8,9], which play a pivotal role in physiological conditions and inflammatory diseases [10-12]. Accumulating evidence suggests that the expression of miR-297 is frequently altered in many tumors and inflammatory diseases, such as bacterial infections or sepsis [13-17]. In addition, abnormal activation and dysfunction of endothelial cells are required for various vascular inflammatory diseases, such as sepsis, diabetes, rheumatoid arthritis, and inflammatory bowel diseases [18-21]. During inflammation, the activated vascular endothelium expresses various adhesion molecules (VCAM-1, ICAM-1 and E-selectin), which can recruit leukocytes, and regulate leukocyte attachment on the vascular endothelial cells at the early stage [21]. However, the role of miR-297 in inflammatory responses of vascular endothelial cells in sepsis is still unclear.

In the present study, we hypothesized that miR-297 influences the expressions of inflammatory cytokines in LPS-stimulated HUVECs, and is involved in the apoptosis of HUVECs. Firstly, we established a model of inflammatory HUVECs using LPS-induced HUVECs in vitro. Then, we over-expressed miR-297 by transfection of miR-297 mimics into HUVECs. Finally, we investigated the role of miR-297 in LPS-induced inflammatory response and apoptosis using in-vitro assays.

\section{Materials and Methods}

\section{Cell culture and ethics statement}

This study was approved by the Ethics Committee of Shandong Provincial Third Hospital. Written informed consent was obtained from all subjects prior to the study. HUVECs were obtained from five human donors. Umbilical cords (10 cm in length) were resected soon after birth, and immediately stored in phosphate buffered saline (PBS). The HUVECs were obtained from the lumens of human umbilical veins using collagen enzyme solution. Then, cells were cultured in endothelial cell medium (ECM) (ScienCell, United States). HUVECs were incubated at $37^{\circ} \mathrm{C}$ in $5 \% \mathrm{CO}_{2}$ and maintained using standard cell culture. After reaching a confluence of $80 \%$, HUVECs were detached using $0.25 \%$ trypsin-EDTA. Human embryonic kidney293T (HEK-293T) cells (Cell Resource Center of Shanghai Institute of Life Science, Shanghai, China) were cultured in Dulbecco's Modified Eagle Medium (DMEM, Invitrogen, Carlsbad, CA, USA) supplemented with $10 \%$ FBS (Thermo Fisher Scientific, Inc.), $100 \mathrm{U} / \mathrm{mL}$ penicillin (Invitrogen) and $100 \mathrm{mg} / \mathrm{mL}$ streptomycin (Invitrogen, Carlsbad, CA, USA) at $37^{\circ} \mathrm{C}$ in a humidified and $5 \% \mathrm{CO}_{2}$ atmosphere.

\section{Cell transfection}

Negative control miRNA (miR-NC), miR-297 mimics, STAT3-small interfering RNA (si-STAT3), and negative control siRNAs (si-control) were all purchased from GenePharma Co. (Shanghai, China). The full-length STAT3 was ligated into a pcDNA3.1 plasmid (Shanghai GenePharma Co., Ltd, China), and the recombined plasmid was referred to as pcDNA3.1-STAT3. Lipofectamine 2000 regent (Invitrogen, Carlsbad, CA, USA) was utilized for cell transfection in line with the protocol of the manufacturer. The empty pcDNA3.1 plasmid was also transfected into cells, termed as a vector group. Stable transfection was selected by 0.5 $\mathrm{mg} / \mathrm{mL}$ G418 (Sigma-Aldrich, St. Louis, MO, USA). Cells transiently transfected with miRNAs were harvested at $72 \mathrm{~h}$ post-transfection. 


\section{Cellular Physiology Cell Physiol Biochem 2019;52:696-707

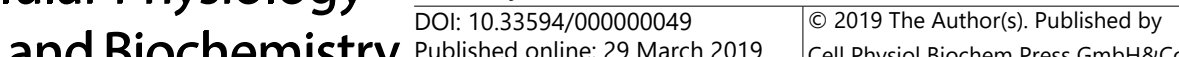 \\ Published online: 29 March 2019 Cell Physiol Biochem Press GmbH\&Co. KG \\ Yao et al.: miR-297 Protects HUVECs}

\section{Western blot}

Briefly, cells were lysed with RIPA lysis buffer in the presence of protease inhibitor cocktail (Merck, Darmstadt, Germany). Cell debris was removed by centrifugation at $12,000 \mathrm{rpm}$ for $12 \mathrm{~min}$. The protein content was determined with BCA kit and separated by 8 10\% SDS-PAGE, and then transferred to polyvinylidene fluoride (PVDF) membranes (Millipore, Bedford, MA, USA). The membranes were blocked in $5 \%(\mathrm{w} / \mathrm{v})$ non-fat dry milk in Tris-buffered saline (TBS) and $0.1 \%(\mathrm{v} / \mathrm{v}$ ) Tween 20 for $1 \mathrm{~h}$ at room temperature, and then incubated with the primary antibodies $\left(1: 500\right.$; Abcam, Cambridge, MA, USA) at $4{ }^{\circ} \mathrm{C}$ overnight. GAPDH (1:1000; Santa Cruz Biotechnology, Paso Robles, CA, USA) was used as an intern control. After incubation of appropriate secondary horseradish peroxidase-conjugated antibodies including HRPconjugated anti-rabbit or anti-mouse (1:1000; Cell Signaling Technology, Inc., Beverly, MA, USA) for $1 \mathrm{~h}$, blots were visualized with ECL kit. Band intensities were measured by densitometry (Chemi Doc Documentation System/Quantity One quantitation software, Bio-Rad Laboratories Inc, Hercules, CA, USA). Variations in protein levels were expressed as fold change compared with control after normalization to GAPDH.

\section{Quantitative real-time PCR and RT-PCR}

Total RNA was isolated from cells using the TRIzol reagent (Invitrogen, Carlsbad, CA, USA) according to the manufacturer's instructions. An aliquot of $2 \mu \mathrm{g}$ RNAs were reversely transcribed with a PrimeScript RT reagent kit (Bio-Rad Laboratories, Hercules, CA, USA), and real-time PCR was performed using SYBR Green PCR Master Mix (Bio-Rad Laboratories) on a MyiQ Single Color Real-time PCR Detection System (Bio-Rad Laboratories). The sequence-specific primers for the indicated genes were synthesized by Sangon Biothech (Shanghai, China). To detect miRNA expression, reverse transcription was performed using the TaqMan ${ }^{\circledR}$ MicroRNA Reverse Transcription Kit (Applied Biosystems, Carlsbad, CA, USA) and PCR reactions were conducted using the TaqMan ${ }^{\circledR}$ MicroRNA Assay Kit (Applied Biosystems). Primers for miR-297, and the small nuclear RNA U6 were from RiboBio Co., Ltd. (Guangzhou, China). The fold change in expression for each gene was calculated using the $2^{-\Delta \Delta} \mathrm{CT}$ method, with U6 as an internal control. After amplification, $10 \mu \mathrm{l}$ of products were separated by electrophoresis on a $1.5 \%$ agarose gel and visualized by ethidium bromide staining under UV light.

\section{Apoptosis assay}

Quantization of apoptotic cells were performed using Annexin V-FITC/PI apoptosis detection kit (Beijing Biosea Biotechnology, China). In brief, the transfected cells were planted in 6-well plates at a density of $1 \times 10^{6}$ cells per well and were subjected to $100 \mathrm{ng} / \mathrm{mL}$ LPS for $24 \mathrm{~h}$. Cells were then collected and resuspended in $200 \mu \mathrm{L}$ binding buffer (Beijing Biosea Biotechnology, China) containing $10 \mu \mathrm{L}$ Annexin V-FITC. After 30 min incubation in the dark at room temperature, $5 \mu \mathrm{L}$ PI and $300 \mu \mathrm{L}$ binding buffer were added, and the samples were immediately analyzed on a flow cytometer (BD Biosciences, USA). Annexin-V positive cells were recognized as apoptotic cells.

\section{MiRNA target prediction}

The potential target genes of miRNAs were predicted in silico using different miRNA target prediction algorithms: TargetScan (http://www.targetscan.org/), DIANA-MICROT (http://diana.cslab.ece.ntua.gr/ micro-CDS/), miRDB (http://mirdb.org/miRDB/), miRanda (http://www.microrna.org/microrna/), and miRwalk (http://zmf.umm.uni-heidelberg.de/apps/zmf/mirwalk2/).

\section{Dual luciferase reporter assay}

Vectors containing wild-type (Wt) or mutant (Mut) STAT3 3'-UTR were obtained from GenePharma (Shanghai, China) and co-transfected with miR-297 mimics into HEK-293T cells. Forty-eight hours later, HEK-293T cells were lysed. Firefly and Renilla luciferase activities were measured with a Dual-Luciferase Reporter Assay Kit (TransGen Biotech Co., Ltd. China) using a dual luciferase-reporter assay system (Berthold, Germany). The results were evaluated by normalizing the firefly luciferase activity to the Renilla luciferase activity. 


\section{Cellular Physiology Cell Physiol Biochem 2019;52:696-707 \\ \begin{tabular}{ll|l} 
and Biochemistry & $\begin{array}{l}\text { DOl: 10.33594/000000049 } \\
\text { Published online: } 29 \text { March } 2019\end{array}$ & $\begin{array}{l}\text { C) } 2019 \text { The Author(s). Published by } \\
\text { Cell Physiol Biochem Press GmbH\&Co. KG }\end{array}$
\end{tabular} \\ Yao et al.: miR-297 Protects HUVECs}

Statistical analysis

Data are presented as means \pm standard deviation (SD). Comparisons among multiple groups were carried out using the one-way analysis variance (ANOVA) followed by Bonferroni's comparison test. Statistical calculations were performed by GraphPad Prism (GraphPad Software, San Diego, CA, USA). P < 0.05 was considered statistically significant.

\section{Results}

The inflammatory HUVECs model is successfully established by LPS

To generate LPS-induced inflammatory HUVECs model, we firstly explored the rational dosing and the time course of LPS action. HUVECs were administrated with different concentrations of LPS $(0,10,100$, or $1000 \mathrm{ng} / \mathrm{mL})$ at different time points $(0,6,12,24$, and $48 \mathrm{~h}$ ). Then we measured the expression level of miR-297 using RT-PCR and qRT-PCR. Meanwhile, inflammatory cytokines (IL- 6 and TNF- $\alpha$ ) and adhesion molecules (ICAM-1, VCAM-1, and E-selectin) were further detected by Western blot analysis. The results showed that miR-297 expression was significantly down-regulated after LPS treatment in a timeand dose-dependent manner ( $\mathrm{P}<0.001$, Fig. $1 \mathrm{~A})$. In addition, the protein expression levels of STAT3, IL-6, TNF- $\alpha$, ICAM-1, VCAM-1, and E-selectin were markedly increased in a time- and dose-dependent manner upon LPS stimulation $(\mathrm{P}<0.001$, Fig. 1A). Notably, when HUVECs were subjected to $100 \mathrm{ng} / \mathrm{mL}$ LPS for $24 \mathrm{~h}$, miR-297 expression decreased by approximately $50 \%$, while the expressions of inflammatory cytokines and adhesion molecules increased by approximately $56 \%$. These findings indicated the inflammatory HUVECs model was successfully established after stimulation for $24 \mathrm{~h}$ with $100 \mathrm{ng} / \mathrm{mL}$ LPS.

\section{LPS induces apoptosis of HUVECS}

To figure out the role of LPS in apoptosis of HUVECs, AnnexinV-FITC/PI staining was used to detect apoptosis, and Western blot analysis was used to measure the expression levels of apoptosis-related proteins in inflammatory HUVECs model. We found that the percentage of apoptotic cells was markedly elevated, accompanied with the increase of LPS concentration (Fig. 1B), indicating cell viability was severely affected. Compared with HUVECs treated with $0 \mathrm{ng} / \mathrm{mL}$ LPS, the difference of cell apoptosis in LPS-induced HUVECs was statistically significant $(\mathrm{P}<0.001)$. Similarly, Bcl-2 expression was significantly downregulated, while the expressions of Bax and cleaved caspase- 3 were gradually up-regulated with the increase of LPS concentration ( $P<0.001$, Fig. 1C). These findings suggested that LPS induced apoptosis of inflammatory HUVECs.

miR-297 mimics increase miR-297 expression in HUVECs

In this work, we further investigated how miR-297 affected cell apoptosis and expressions of inflammatory cytokines. Because miR-297 was down-regulated in LPS-induced HUVECs, miR-297 mimics were transfected into HUVECs to increase the expression of miR-297. Then, HUVECs were administrated in the presence or absence of $100 \mathrm{ng} / \mathrm{mL}$ LPS for $24 \mathrm{~h}$. The qRTPCR analysis revealed that miR-297 expression in HUVECs was markedly up-regulated after transfection with miR-297 mimics compared with miR-NC ( $<<0.001$, Fig. $2 \mathrm{~A})$.

\section{LPS-induced inflammatory injury is alleviated by miR-297 over-expression}

Firstly, we investigated the effect of miR-297 over-expression on the expressions of inflammatory cytokines. HUVECs with miR-297 mimics were administrated in the presence or absence of $100 \mathrm{ng} / \mathrm{mL}$ LPS for $24 \mathrm{~h}$. Expressions of inflammatory cytokines were detected by Western blot assay. We found that miR-297 over-expression obviously inhibited LPSinduced expressions of STAT3, IL-6, TNF- $\alpha$, ICAM-1, VCAM-1 and E-selectin ( $<<0.001$, Fig. 2B). At the same time, negative associations were confirmed among miR-297 and inflammatory cytokines, suggesting miR-297 may play an important role in suppressing inflammatory responses of LPS-induced HUVECs. Additionally, apoptosis assay showed that 


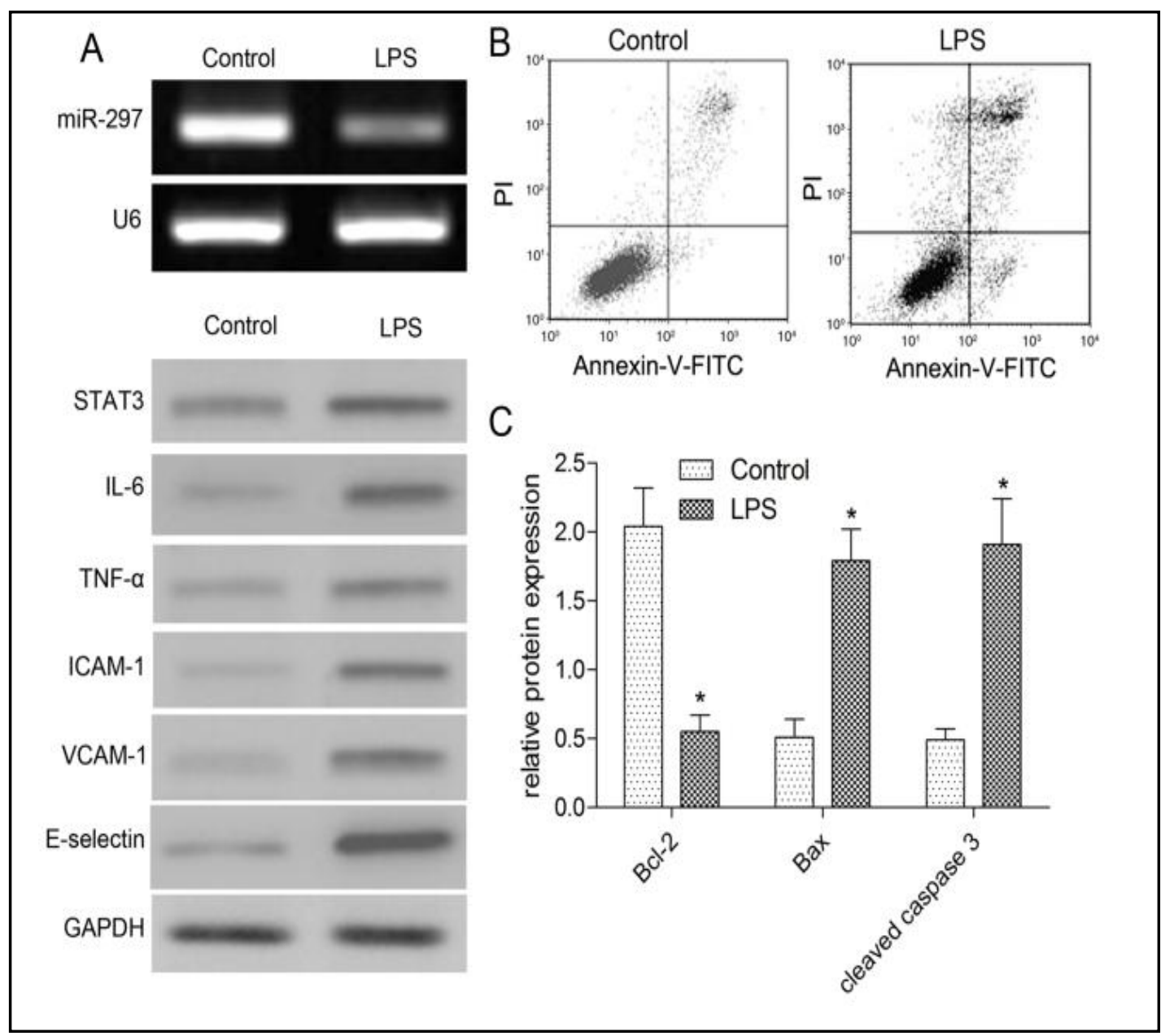

Fig. 1. LPS induces inflammatory response and apoptosis in HUVECs. (A) HUVECs were incubated with different concentrations of LPS at different time points. MiR-297 expression was detected with RTPCR and normalized to endogenous U6. STAT3, inflammatory cytokines (IL-6 and TNF- $\alpha$ ), and adhesion molecules (ICAM-1, VCAM-1 and E-selectin) were determined by Western blot analysis. Band intensities were measured by densitometry. Variations in protein levels were expressed as fold change compared with control after normalization to GAPDH. HUVECs administrated with 0.01M PBS were used as control. (B) The percentage of apoptotic cells was detected by Annexin V-FITC/PI double staining and flow cytometry analysis. (C) Apoptosis-related proteins were measured by Western blot. Each experiment was performed in triplicate independently. Asterisks indicated statistically significant differences. Data was represented as means \pm SD. $*$ P $<0.001$, v.s. control.

the percentage of apoptotic cells of HUVECs was significantly increased at $24 \mathrm{~h}$ following stimulation with $100 \mathrm{ng} / \mathrm{mL}$ LPS. By contrast, the percentage of apoptotic cells of HUVECs transfected with miR-297 mimics was significantly decreased (P < 0.01, Fig. 2C), indicating that miR-297 had a protective effect on HUVECs. Finally, we demonstrated that Bax and cleaved caspase- 3 proteins were highly expressed in LPS-induced HUVECs, which were subsequently suppressed by miR-297 mimics ( $\mathrm{P}<0.001$, Fig. 2D). However, Bcl- 2 protein level was reduced by LPS, which was subsequently increased after transfection with miR297 mimics ( $P<0.001$, Fig. 2D). These results suggested miR-297 protected HUVECs against LPS-induced inflammatory injury. 


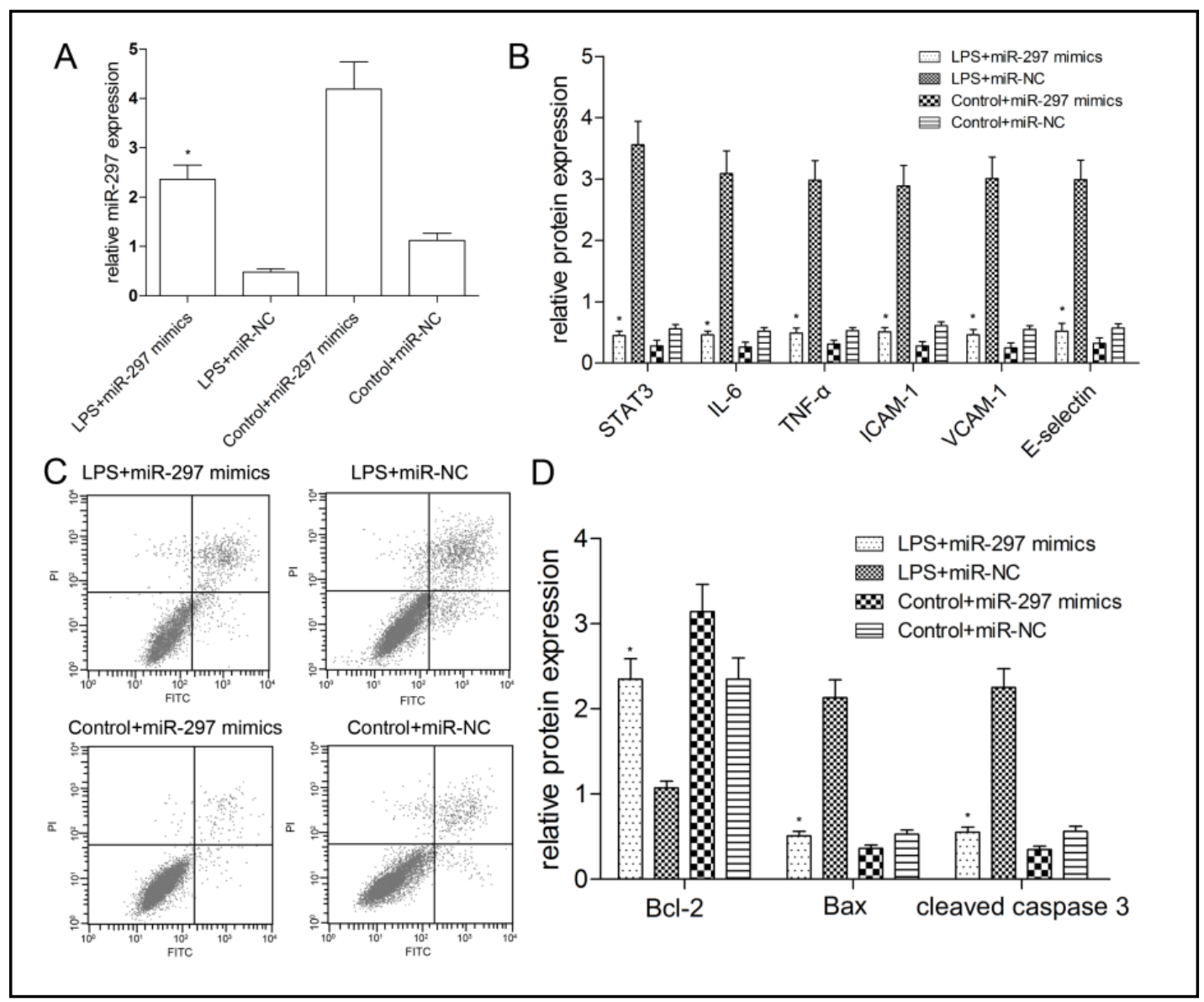

Fig. 2. MiR-297 alleviates LPS-induced inflammatory response and apoptosis of HUVECs. HUVECs were transfected with miR-297 mimics or miR-NC, and then administrated with $100 \mathrm{ng} / \mathrm{mL}$ LPS for $24 \mathrm{~h}$. (A) The expression of miR-297 was measured by qRT-PCR. (B) STAT3, inflammatory cytokines (IL-6 and TNF- $\alpha$ ), and adhesion molecules (ICAM-1, VCAM-1 and E-selectin) were determined by Western blot analysis. GAPDH was used as an internal control. (C) The percentage of apoptotic cells was detected by Annexin V-FITC/ PI double staining and flow cytometry analysis. (D) Apoptosis-related factors were measured by Western blot. Each experiment was performed in triplicate independently. Asterisks indicated statistically significant differences. Data was represented as means \pm SD. $* \mathrm{P}<0.001$, v.s. control.

\section{STAT3 is a direct target of miR-297}

TargetScan and miRanda algorithms predicted that the transcriptional STAT3 was a candidate target of miR-297. Then we performed Western blot and dual luciferase reporter assay to identify whether the 3'-UTR sequences of STAT3 mRNA were targeted by miR297. Two plasmids were generated by cloning the respective fragments from the $3^{\prime}$-UTR of STAT3 into the downstream region of the Renilla luciferase reporter gene. HEK-293T cells were then co-transfected with one of the wild type or mutant reporter vectors and miR-297 mimics or miR-NC. Western blot analysis revealed that the expression of STAT3 protein was obviously down-regulated in HEK-293T cells co-transfected with wild-type STAT3 3'-UTR and miR-297 mimics ( $\mathrm{P}<0.001$, Fig. 3A and B). The dual luciferase reporter assay validated that the luciferase activity of the wild-type was inhibited by miR-297 mimics as compared with miR-NC ( $<<0.01$, Fig. $3 C)$, while there were no significant differences in the luciferase activity of the mutant type between miR-297 mimics and miR-NC group (P > 0.05, Fig. 3D). These results indicated that STAT3 was a direct target of miR-297. 


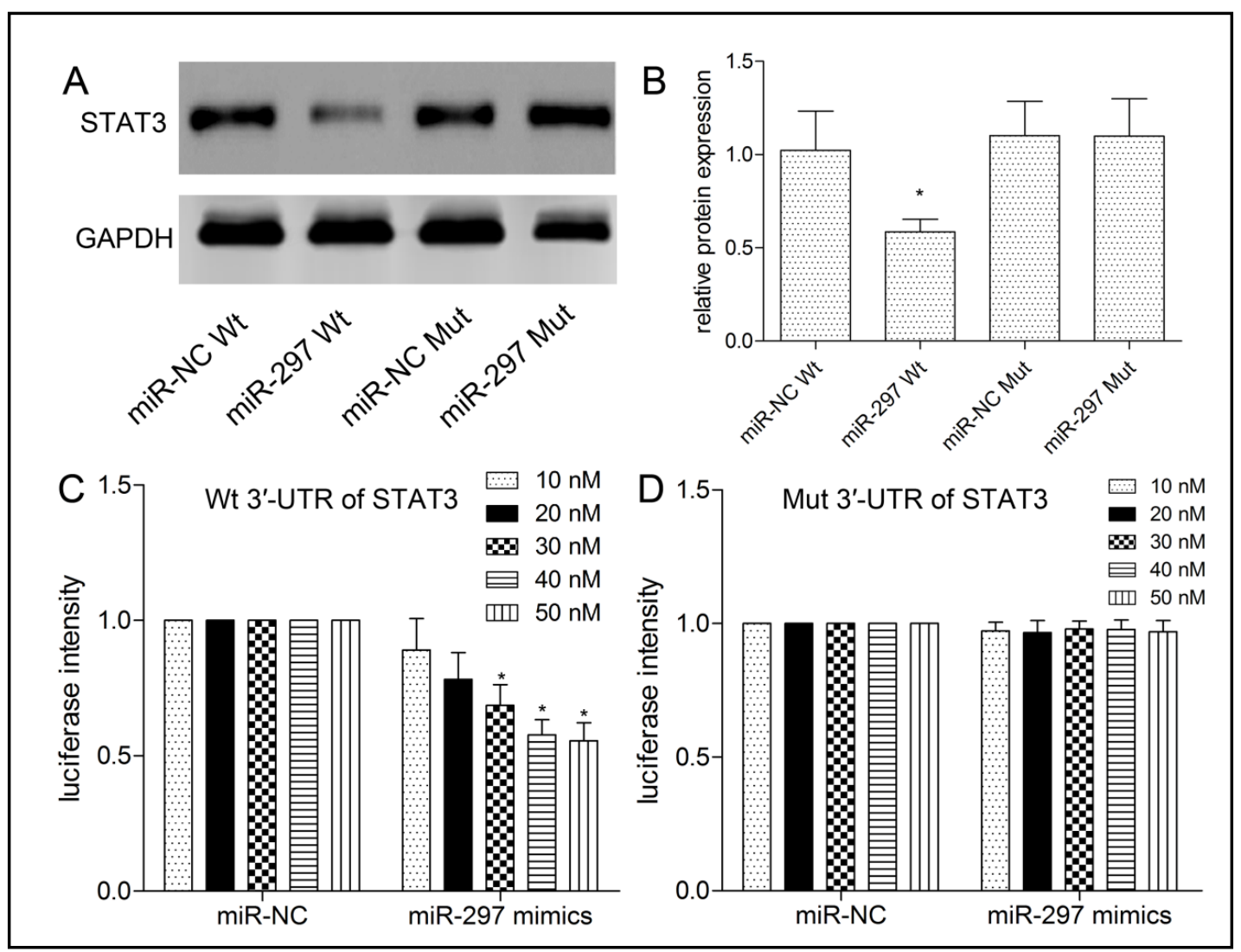

Fig. 3. MiR-297 directly targets the 3'-UTR of STAT3 mRNA. (A) Expression of STAT3 in HEK-293T cells co-transfected with miR-297 mimics or miR-NC, and STAT3 vector containing the Wt or Mut 3'-UTR was assessed by Western blot. (B) Band intensities were measured by densitometry. Variations in protein levels were expressed as fold change compared with control after normalization to GAPDH. (C, D) The wild and mutant types of $3^{\prime}$-UTR of STAT3 mRNA were constructed in the reporter vector, then co-transfected with miR-297 mimics into HEK-293T cells, and finally tested by a double luciferase reporter gene system. Each experiment was performed in triplicate independently. Asterisks indicated statistically significant differences. Data was represented as means \pm SD. $* \mathrm{P}<0.001$, v.s. control.

\section{LPS-induced inflammatory injury is exacerbated by restored STAT3 expression}

In order to explore the effect of STAT3 on LPS-induced inflammatory injury in HUVECs, the pcDNA3.1-STAT3 plasmid or pcDNA3.1 vector were co-transfected into HUVECs with miR-297 mimics. The percentage of apoptotic cells was determined by flow cytometry. The effects of STAT3 on inflammatory cytokines and adhesion molecules in LPS-induced HUVECs were evaluated by Western blot analysis. The results revealed the protein levels of IL6 , TNF- $\alpha$, ICAM-1, VCAM-1 and E-selectin in STAT3-overexpressed HUVECs were drastically up-regulated compared with their respective controls ( $\mathrm{P}<0.01$, Fig. 4A). Over-expression of STAT3 restored the expressions of miR-297 mimics-inhibited STAT3, IL-6, TNF- $\alpha$, ICAM1, VCAM-1 and E-selectin. In addition, the percentage of apoptotic cells was significantly increased in STAT3-overexpressed HUVECs than that in LPS alone-treated group. Overexpression of STAT3 markedly increased miR-297 mimics-repressed apoptotic rate (P < 0.01 , Fig. 4B). To further confirm our results at a molecular level, we performed Western blot to detect the protein expression levels of apoptosis-related biomarkers, including Bax, Bcl-2 and cleaved caspase-3. As illustrated in Fig. 4C, the expressions of Bax and cleaved caspase-3 proteins in STAT3-overexpressed HUVECs were obviously up-regulated compared with LPS alone-treated group, whereas this promotion effect was rapidly inhibited by cotransfection with miR-297 mimics $(\mathrm{P}<0.001)$. In addition, the expression level of Bcl-2 


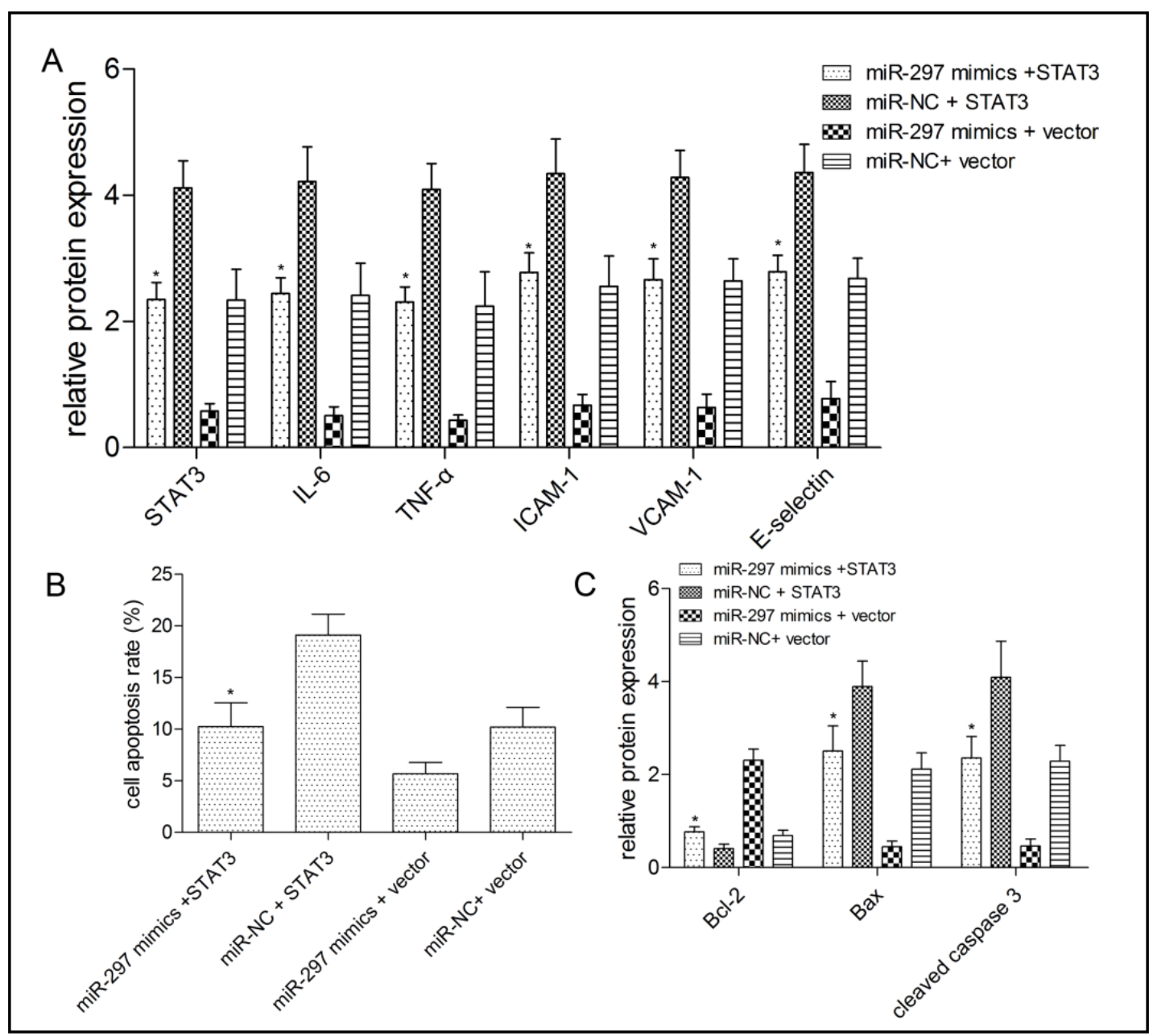

Fig. 4. Restored STAT3 expression inhibits the protective effects of miR-297 on HUVECs. (A) HUVECs were co-transfected with miR-297 mimics and pcDNA3.1-STAT3. After administration of $100 \mathrm{ng} / \mathrm{mL}$ LPS for 24 $\mathrm{h}$, all proteins were measured using Western blot analysis. (B) Apoptosis was detected by flow cytometric analysis. (C) The percentage of apoptotic cells was detected by Annexin V-FITC/PI double staining and flow cytometry analysis. Each experiment was performed in triplicate independently. Asterisks indicated statistically significant differences. Data was represented as means \pm SD. ${ }^{*} \mathrm{P}<0.001$, v.s. control.

was down-regulated by STAT3 over-expression and subsequently up-regulated after cotransfection with miR-297 mimics. These data indicated that LPS-induced inflammatory injury was exacerbated by restored STAT3 expression.

\section{Suppression of STAT3 contributes to the protective effects of miR-297 on HUVECs}

To confirm if miR-297 exert its biological functions by targeting STAT3, we co-transfected si-STAT3 and miR-297 mimics into HUVECs. Then HUVECs were administrated with 100 ng/mL LPS for 24 hours. Firstly we demonstrated that STAT3 protein level was indeed decreased by si-STAT3 compared with si-control (P < 0.001, Fig. 5A). Then, Annexin V-FITC/ PI apoptosis assay revealed that cell apoptosis was significantly alleviated by co-transfection of si-STAT3 and miR-297 mimics, compared with three other groups $(\mathrm{P}<0.001)$, indicating cell viability may be restored by suppression of STAT3. Further, co-transfection of si-STAT3 and miR-297 mimics obviously inhibited the expressions of inflammatory cytokines and adhesion molecules compared with si-STAT3 alone or miR-297 mimics alone ( $P<0.001$, Fig. 5A). Finally, we identified that co-transfection of si-STAT3 and miR-297 mimics obviously inhibited the expressions of Bax and cleaved caspase-3, and markedly up-regulated the 


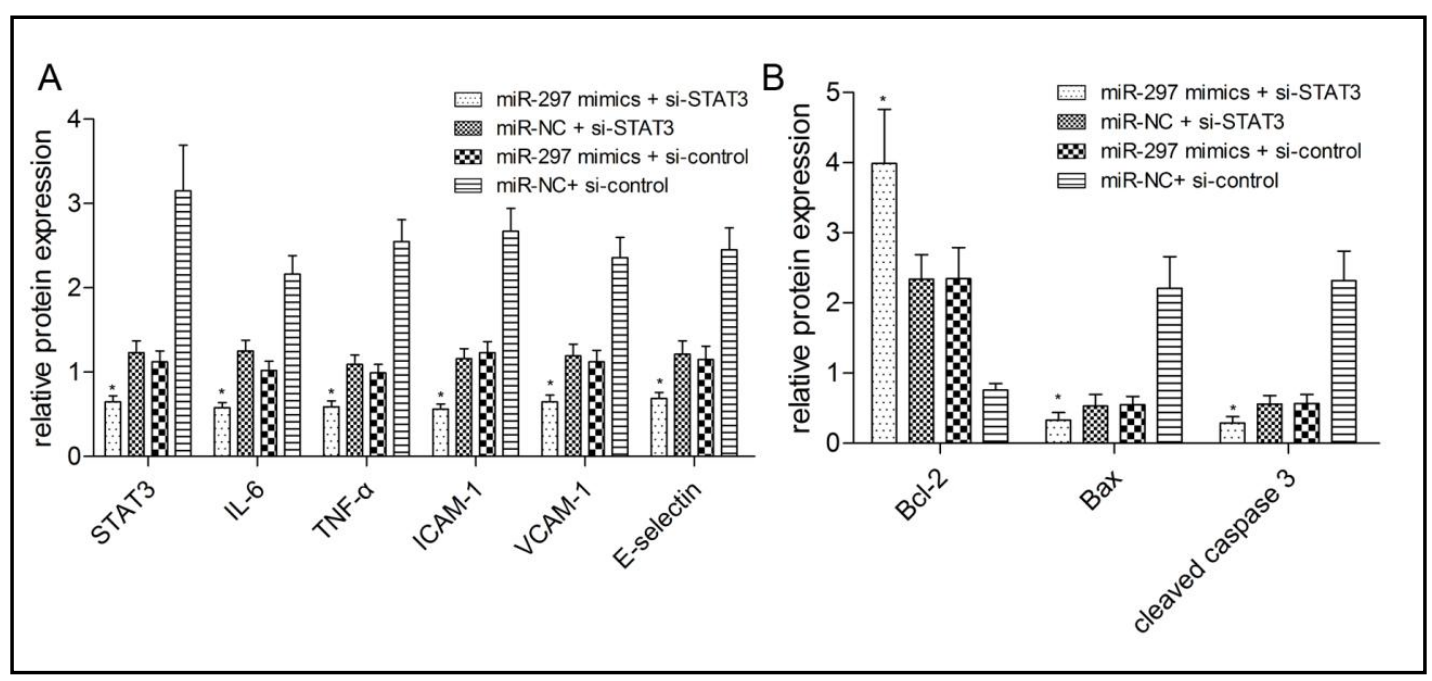

Fig. 5. Suppression of STAT3 contributes to the protective effects of miR-297 on HUVECs. (A) HUVECs were cotransfected with miR-297 mimics and si-STAT3. After administration of $100 \mathrm{ng} / \mathrm{mL}$ LPS for $24 \mathrm{~h}$, all proteins were measured by Western blot. (B) Apoptosis-related proteins were measured by Western blot. Each experiment was performed in triplicate independently. Asterisks indicated statistically significant differences. Data was represented as means \pm SD. $*$ P $<0.001$, v.s. control.

expression of Bcl-2 compared with three other groups ( $\mathrm{P}<0.001$, Fig. 5B). Our findings indicated that suppression of STAT3 contributed to the protective effects of miR-297 on HUVECs.

\section{Discussion}

To date, it has been reported that activation of endothelial cells and exposure to endotoxins lead to the development of sepsis. LPS, also known as lipoglycans and endotoxins, is released from bacterial membranes, which binds to the endothelial cell surface, and triggers a potent inflammatory responses [22, 23]. Recently, miRNAs have been demonstrated to play a crucial role in LPS-mediated inflammatory factors. Although miR-297 is associated with LPS-induced inflammatory processes, and involved in the development of sepsis [24], it is not clear how miR-297 modulates inflammatory responses in LPS-induced vascular endothelial cells.

In previous studies, LPS can activate STAT3 signaling via protein phosphorylation [23]. Once activated, phosphorylated STAT3 dimerizes and enters the nucleus to activate transcription of various genes [25]. Currently, STAT3 signaling has been shown to be activated in inflammatory vascular endothelial cells, and induced the expressions of inflammatory cytokines [26-28]. Once STAT3 is persistently activated, septic diseases will occur [29]. Till now, STAT3-involved pathway in sepsis is still unknown. In this work, we explored the potential molecular mechanisms of miR-297 and STAT3 using LPS-induced inflammatory HUVECs model. We found that miR-297 over-expression decreased expressions of LPSinduced STAT3, inflammatory cytokines and adhesion molecules, and inhibited LPSinduced apoptosis of HUVECs. Besides, apoptosis-related proteins induced by LPS were also reversed by miR-297 over-expression. Considering that STAT3 is closely related to T and $B$ cell activation, immune responses and inflammatory reaction [30], we assumed that miR-297 may protect HUVECs against LPS-induced inflammatory response and apoptosis through inhibition of STAT3.

MiRNAs achieve their biological function through binding the 3'-UTR of target genes and regulating the expression levels of protein-coding genes. Recent studies showed that some miRNAs participated in regulation of STAT3 pathway. Luo Y et al. reported that miR- 


\section{Cellular Physiology Cell Physiol Biochem 2019;52:696-707

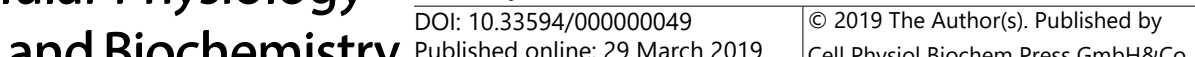 \\ Published online: 29 March 2019 Cell Physiol Biochem Press GmbH\&Co. KG \\ Yao et al.: miR-297 Protects HUVECs}

20b negatively regulated VEGF expression by targeting STAT3 in hepatocellular carcinoma cells [31]. Cheng DL et al. suggested that miR-34a promoted iNOS secretion from pulmonary macrophages in septic suckling rats through activating STAT3 pathway [32]. Zhang W et al. indicated that overexpression of miR-4443 promoted the resistance of non-small cell lung cancer cells to epirubicin by targeting INPP4A, and regulating activation of JAK2/ STAT3 pathway [33]. In this study, we demonstrated that miR-297 reduced the expression of STAT3 protein by targeting its 3'-UTR. Meanwhile, miR-297-inhibited inflammatory cytokines, adhesion molecules and apoptosis were further repressed by silencing of STAT3. On the contrary, LPS-induced cell injury was exacerbated by restored STAT3 expression. These findings suggested that LPS/miR-297/STAT3 pathways may play a crucial role in inflammatory processes of HUVECs.

\section{Conclusion}

In conclusion, our study suggested that miR-297 inhibited LPS-induced inflammatory response and apoptosis of HUVECs by regulating STAT3 signaling pathway. These findings may help us understand the pathogenesis of sepsis, and provide a novel therapeutic approach for prevention and/or treatment of sepsis.

\section{Acknowledgements}

We are thankful to doctors and nurses in the ICU for their great support.

\section{Disclosure Statement}

The authors have declared that no conflict of interest exists.

\section{References}

1 Kumar S, Tripathy S, Jyoti A, Singh SG: Recent advances in biosensors for diagnosis and detection of sepsis: A comprehensive review. Biosens Bioelectron 201;124-125:205-215.

$>2$ O’Brien Z, Cass A, Cole L, Finfer S, Gallagher M, McArthur C, McGuiness S, Myburgh J, Bellomo R, Mårtensson J: RENAL Study Investigators and the Australian and New Zealand Intensive Care Clinical Trials Group: Sex and mortality in septic severe acute kidney injury. J Crit Care 2018;49:70-76.

- 3 Sertaridou EN, Chouvarda IG, Arvanitidis KI, Filidou EK, Kolios GC, Pnevmatikos IN, Papaioannou VE: Melatonin and cortisol exhibit different circadian rhythm profiles during septic shock depending on timing of onset: a prospective observational study. Ann Intensive Care 2018;8:118.

- 4 Bomans K, Schenz J, Sztwiertnia I, Schaack D, Weigand MA, Uhle F: Sepsis Induces a Long-Lasting State of Trained Immunity in Bone Marrow Monocytes. Front Immunol 2018;9:2685.

5 Sousa-Victor P, Jasper H, Neves J: Trophic Factors in Inflammation and Regeneration: The Role of MANF and CDNF. Front Physiol 2018;9:1629.

- 6 Salybekov AA, Kawaguchi AT, Masuda H, Vorateera K, Okada C, Asahara T: Regeneration-associated cells improve recovery from myocardial infarction through enhanced vasculogenesis, anti-inflammation, and cardiomyogenesis. PLoS One 2018;13:e0203244.

7 Lee ES, Kwon MH, Kim HM, Kim N, Kim YM, Kim HS, Lee EY, Chung CH: Dibenzoylmethane ameliorates lipid-induced inflammation and oxidative injury in diabetic nephropathy. J Endocrinol 2019;240:169-179. Liu J, Wang HZ, Sun Y: Inhibition of CXCR4 by MicroRNA-1192 Reduces the Activation of Th17 Cells and Expression of Inflammation Factors in a Mouse Model of Vulvovaginal Candidiasis. Cell Physiol Biochem 2018;50:893-910. 


\section{Cellular Physiology Cell Physiol Biochem 2019;52:696-707 \begin{tabular}{ll|l|l} 
DOI: 10.33594/000000049 & (O) 2019 The Author(s). Published by \\
\hline
\end{tabular} and BiOchemistry Published online: 29 March 2019 Cell Physiol Biochem Press GmbH\&Co. KG \\ Yao et al.: miR-297 Protects HUVECs}

9 Rovira-Llopis S, Escribano-Lopez I, Diaz-Morales N, Iannantuoni F, Lopez-Domenech S, Andújar I, Jover A, Pantoja J, Pallardo LM, Bañuls C, Victor VM: Downregulation of miR-31 in Diabetic Nephropathy and its Relationship with Inflammation. Cell Physiol Biochem 2018;50:1005-1014.

- 10 Yang Y, Liu D, Xi Y, Li J, Liu B, Li J: Upregulation of miRNA-140-5p inhibits inflammatory cytokines in acute lung injury through the MyD88/STAT3 signaling pathway by targeting TLR4. Exp Ther Med. 2018;16:39133920.

11 Ma J, Chen X, Xin G, Li X: Chronic exposure to the ionic liquid [C8mim]Br induces inflammation in silver carp spleen: Involvement of oxidative stress-mediated p38MAPK/STAT3 signalling and microRNAs. Fish Shellfish Immunol 2018;84:627-638.

12 Ma Y, Liu Y, Hou H, Yao Y, Meng H: miR-150 predicts survival in patients with sepsis and inhibits LPSinduced inflammatory factors and apoptosis by targeting NF- $\kappa$ B1 in human umbilical vein endothelial cells. Biochem Biophys Res Commun 2018;500:828-837.

13 Zhang CZ: Long intergenic non-coding RNA 668 regulates VEGFA signaling through inhibition of miR-297 in oral squamous cell carcinoma. Biochem Biophys Res Commun 2017;489:404-412.

14 Bao Q Zhao M, Chen L, Wang Y, Wu S, Wu W, Liu X: MicroRNA-297 promotes cardiomyocyte hypertrophy via targeting sigma-1 receptor. Life Sci 2017;175:1-10.

15 Sun Y, Zhao J, Yin X, Yuan X, Guo J, Bi J: miR-297 acts as an oncogene by targeting GPC5 in lung adenocarcinoma. Cell Prolif 2016;49:636-643.

- 16 Kefas B, Floyd DH, Comeau L, Frisbee A, Dominguez C, Dipierro CG, Guessous F, Abounader R, Purow B: A miR-297/hypoxia/DGK- $\alpha$ axis regulating glioblastoma survival. Neuro Oncol 2013;15:1652-1663.

- 17 Xu K, Liang X, Shen K, Cui D, Zheng Y, Xu J, Fan Z, Qiu Y, Li Q, Ni L, Liu J: miR-297 modulates multidrug resistance in human colorectal carcinoma by down-regulating MRP-2. Biochem J 2012;446:291-300.

18 Zhou M, Ding Y, Cai L, Wang Y, Lin C, Shi Z: Low molecular weight fucoidan attenuates experimental abdominal aortic aneurysm through interfering the leukocyte-endothelial cells interaction. Mol Med Rep 2018;17:7089-7096.

19 Dickinson CM, LeBlanc BW, Edhi MM, Heffernan DS, Faridi MH, Gupta V, Cioffi WG, O’Brien X, Reichner JS: Leukadherin-1 ameliorates endothelial barrier damage mediated by neutrophils from critically ill patients. J Intensive Care 2018;6:19.

20 Wang Y, Che J, Zhao H, Tang J, Shi G: Osthole alleviates oxidized low-density lipoprotein-induced vascular endothelial injury through suppression of transforming growth factor- $\beta 1 /$ Smad pathway. Int Immunopharmacol 2018;65:373-381.

21 Tettey CO, Yang I, Shin HM: Smilax china leaf extracts suppress pro-inflammatory adhesion response in human umbilical vein endothelial cells and proliferation of HeLa cells. Arch Physiol Biochem 2018;30:1-5.

22 Fang H, Liu A, Chen X, Cheng W, Dirsch O, Dahmen U: The severity of LPS induced inflammatory injury is negatively associated with the functional liver mass after LPS injection in rat model. J Inflamm (Lond) 2018;15:21.

23 Li K, Lv G, Pan L: Sirt1 alleviates LPS induced inflammation of periodontal ligament fibroblasts via downregulation of TLR4. Int J Biol Macromol 2018;119:249-254.

24 Sari AN, Korkmaz B, Serin MS, Kacan M, Unsal D, Buharalioglu CK, Sahan Firat S, Manthati VL, Falck JR, Malik KU, Tunctan B: Effects of 5, 14-HEDGE, a 20-HETE mimetic, on lipopolysaccharide-induced changes in MyD88/TAK1/IKK $\beta / \mathrm{I \kappa B}-\alpha / \mathrm{NF}-\kappa B$ pathway and circulating miR-150, miR-223, and miR-297 levels in a rat model of septic shock. Inflamm Res 2014;63:741-756.

25 Cheng DL, Fang HX, Liang Y, Zhao Y, Shi CS: MicroRNA-34a promotes iNOS secretion from pulmonary macrophages in septic suckling rats through activating STAT3 pathway. Biomed Pharmacother 2018;105:1276-1282.

26 Samanta S, Zhou Z, Rajasingh S, Panda A, Sampath V, Rajasingh J: DNMT and HDAC inhibitors together abrogate endotoxemia mediated macrophage death by STAT3-JMJD3 signaling. Int J Biochem Cell Biol 2018;102:117-127.

- 27 Song JM, Upadhyaya P, Kassie F: Nitric oxide-donating aspirin (NO-Aspirin) suppresses lung tumorigenesis in vitro and in vivo and these effects are associated with modulation of the EGFR signaling pathway. Carcinogenesis 2018;39:911-920.

28 Seo HY, Kim MK, Lee SH, Hwang JS, Park KG, Jang BK: Kahweol Ameliorates the Liver Inflammation through the Inhibition of STAT3 and STAT3 Activation in Primary Kupffer Cells and Primary Hepatocytes. Nutrients 2018;10:E863. 
29 Jiang YX, Dai YY, Pan YF, Wu XM, Yang Y, Bian K, Zhang DD: Total Flavonoids from Radix Glycyrrhiza Exert Anti-Inflammatory and Antitumorigenic Effects by Inactivating iNOS Signaling Pathways. Evid Based Complement Alternat Med 2018;2018:6714282.

30 Zhou Y, Xia L, Liu Q, Wang H, Lin J, Oyang L, Chen X, Luo X, Tan S, Tian Y, Su M, Wang Y, Chen P, Wu Y, Wang H, Liao Q: Induction of Pro-Inflammatory Response via Activated Macrophage-Mediated STAT3 and STAT3 Pathways in Gastric Cancer Cells. Cell Physiol Biochem 2018;47:1399-1410.

31 Luo Y, He J, Tao X, Wang H, Fang Q, Guo S, Song C: miR-20b negatively regulates VEGF expression by targeting STAT3 in H22 hepatocellular carcinoma cells. Oncol Rep 2018;40:2806-2813.

32 Cheng DL, Fang HX, Liang Y, Zhao Y, Shi CS: MicroRNA-34a promotes iNOS secretion from pulmonary macrophages in septic suckling rats through activating STAT3 pathway. Biomed Pharmacother 2018;105:1276-1282.

33 Zhang W, Qiao B, Fan J: Overexpression of miR-4443 promotes the resistance of non-small cell lung cancer cells to epirubicin by targeting INPP4A and regulating the activation of JAK2/STAT3 pathway. Pharmazie 2018;73:386-392. 